\title{
L’allocation des interconnexions par RTE : état des lieux et perspectives
}

\author{
Jean-Philippe Paul et Jean-Yves Bourmaud, RTE
}

\begin{abstract}
Cet article rappelle quelques fondamentaux qui structurent l'accès aux interconnexions sur le réseau de grand transport européen, puis décrit les services d'accès à ces interconnexions qui ont été mis en place par RTE pour les acteurs importateurs/exportateurs. Il explicite les principaux avantages et limites des différents mécanismes, administrés ou de marchés, permettant d'allouer les capacités d'interconnexions lorsqu'elles sont congestionnées.
\end{abstract}

\section{INTRODUCTION}

Nous nous proposons dans cet article de rappeler quelques fondamentaux qui structurent l'accès aux interconnexions sur le réseau de grand transport européen, puis de décrire les services d'accès à ces interconnexions qui ont été mis en place par RTE pour les acteurs importateurs/exportateurs. Ces services s’appuient sur des mécanismes, au départ administrés, aujourd'hui de marchés, permettant de gérer au mieux, dans l'intérêt de l'ensemble des consommateurs, l'accès aux ressources rares que constituent les interconnexions. Parmi ces mécanismes de marchés, nous illustrons certains avantages apportés par un des mécanismes les plus avancés, à savoir le couplage implicite de marchés.

\section{II.QUELQUES ELEMENTS FONDAMENTAUX RELATIFS A L'ACCES AUX INTERCONNEXIONS}

\section{A. La demande d'échanges aux frontières butte sur les capacités}

Considérés de façon globale, les échanges commerciaux entre zones de marché vont des prix les plus bas vers les prix les plus élevés. L'augmentation d'un échange entre deux zones a tendance à faire baisser le prix de la zone de destination et monter celui de la zone d'origine. Si les échanges entre pays européens n'étaient pas limités par des contraintes physiques, les prix de livraison physique auraient tendance à s'égaliser sous l'effet des arbitrages économiques permanents opérés par les acteurs, traders en particulier. L'augmentation des échanges transfrontaliers doit permettre :

Jean-Philippe Paul, RTE Département Méthodes et Appui, Chef du Groupe "Systèmes Electriques et Marchés”. jean-philippe.paul@rte-france.com

Jean-Yves Bourmaud, RTE Département Méthodes et Appui, ingénieur du Groupe "Systèmes Electriques et Marchés" jean-yves.bourmaud@rtefrance.com
D'accroître, dans chaque pays, la concurrence sur les marchés de l'électricité par augmentation du nombre d'acteurs,

De parvenir à une optimisation des ressources au niveau européen, et, de ce fait, faire baisser en moyenne le prix de gros dans l'Union.

Mais, les lignes d'interconnexions européennes, et plus généralement les réseaux à très haute tension qui participent aux échanges transfrontaliers, ont été dimensionnées d'abord pour améliorer la sûreté et la qualité de fourniture, notamment celle des zones frontalières, et pour diminuer les investissements de production nationaux par la mise en commun des réserves (règles UCTE définissant les réserves primaire et secondaire) et l'utilisation du décalage temporel des pointes de consommation, même si des lignes ont également été construites pour permettre la réalisation de contrats de long terme entre pays. Les moyens de production d'électricité actuels étant assez dissemblables d'un pays à un autre, fruit de ressources naturelles et de politiques énergétiques nationales contrastées, le réseau transfrontalier ne peut pas transporter suffisamment de puissance pour suppléer aux différences de prix et permettre à lui tout seul le marché intégré souhaité au niveau européen. C’est l’origine des congestions transfrontalières européennes.

Aux considérations communautaires, s’ajoute la pression des acteurs du marché de gros qui espèrent chacun acheter un peu plus dans les zones de prix bas et vendre un peu plus dans celles de prix haut, et qui se retournent naturellement vers les GRTs dont la mission est de mettre à disposition de leurs clients toutes les ressources du réseau dans les limites de sûreté. Tout cela concourt à solliciter à plein et en permanence toutes les ressources du réseau qui conditionnent le niveau d'échanges transfrontaliers, à commencer par les interconnexions elles-mêmes.

Le réseau européen étant de plus dense et maillé, chacun des échanges commerciaux transfrontaliers contribue aux flux physiques non seulement sur la frontière directement concernée, mais aussi à des degrés divers sur toutes les autres liaisons électriques. En Europe continentale, le flux physique sur chaque interconnexion est ainsi influencé par chacun des échanges commerciaux transfrontaliers.

Le respect de la sûreté du réseau européen impose naturellement de maintenir les flux dans les ouvrages disponibles au-dessous des limites physiques qui s’appliquent à ceux-ci, mais aussi de se prémunir de la perte d'un quelconque d'entre eux (règle du « N-1 »). Du fait du maillage 
des réseaux en Europe continentale, un nombre limité de congestions actives à un instant donné contraint l'ensemble des échanges transfrontaliers. Ainsi par exemple, les limites physiques du réseau influençant principalement la frontière franco-belge contraignent aussi les échanges FranceAllemagne, France-Suisse, Pays-Bas-Belgique, AllemagnePays-Bas, etc ... car chacun d'entre eux contribue aux flux physiques limités. Et dans cet exemple nous n'avons cité que les échanges les plus influents. Il en va de même pour chacun des corridors congestionnés sensibles au commerce transfrontalier, souvent localisés au niveau des lignes frontières mais incluant aussi certains corridors intérieurs.

\section{B. Les besoins d'échanges sont volatils}

$\mathrm{Au}$ fur et à mesure que le marché européen s'ouvre, les différences de prix entre zones de marché deviennent de plus en plus volatiles, se modifiant dans le temps tant en amplitude qu'en direction. La composante fondamentale du prix de chaque zone a beau rester en moyenne relativement stable le prix de livraison physique peut fluctuer de quelques Euros à quelques dizaines d'Euros par MWh autour de ces fondamentaux. Plusieurs raisons contribuent à expliquer cette volatilité. Tout d'abord, les acteurs ont progressé dans l'optimisation de la couverture de leurs fournitures, mettant à profit au mieux les différences de tension des marchés sur des périodes courtes, de l'ordre de l'heure, liées au décalage des courbes de charge ou aux contraintes physiques d'exploitation des différents parcs de production. Ensuite, avec la tendance générale à la réduction de la marge globale de capacité de production, il suffit parfois dans un pays qu'un aléa un peu particulier ou inattendu se réalise -température, nébulosité, sécheresse, indisponibilités fortuites d'unités de productionpour que son prix, au moins pour les pointes journalières ne soit tiré fortement à la hausse. A cela s'ajoutent les incertitudes, les anticipations et les retournements brutaux propres à tout marché ouvert.

Ces fluctuations ne vont pas forcément dans le même sens entre pays voisins : le prix de livraison physique français sera par exemple tantôt inférieur et tantôt supérieur aux prix allemands et anglais. Or ce sont ces différences de prix de livraison physique entre pays voisins, fréquemment variables, qui nourrissent la demande d'échanges transfrontaliers dans des sens différents. En atteste le graphique ci-dessous concernant les échanges commerciaux nets entre France et Belgique et Allemagne pour 2005, où l'on constate des variations de plusieurs milliers de MW en quelques jours.

Dès lors les congestions sensibles au commerce transfrontalier sont activées ou désactivées à un rythme mouvant et la direction des échanges et leurs volumes connaissent des fluctuations importantes.

Compte tenu des délais et des difficultés environnementales pour construire de nouvelles liaisons à très haute tension, ou pour renouveler le parc de production d'un pays, il est probable que cette situation soit durablement installée, voire même qu'elle s'amplifie.

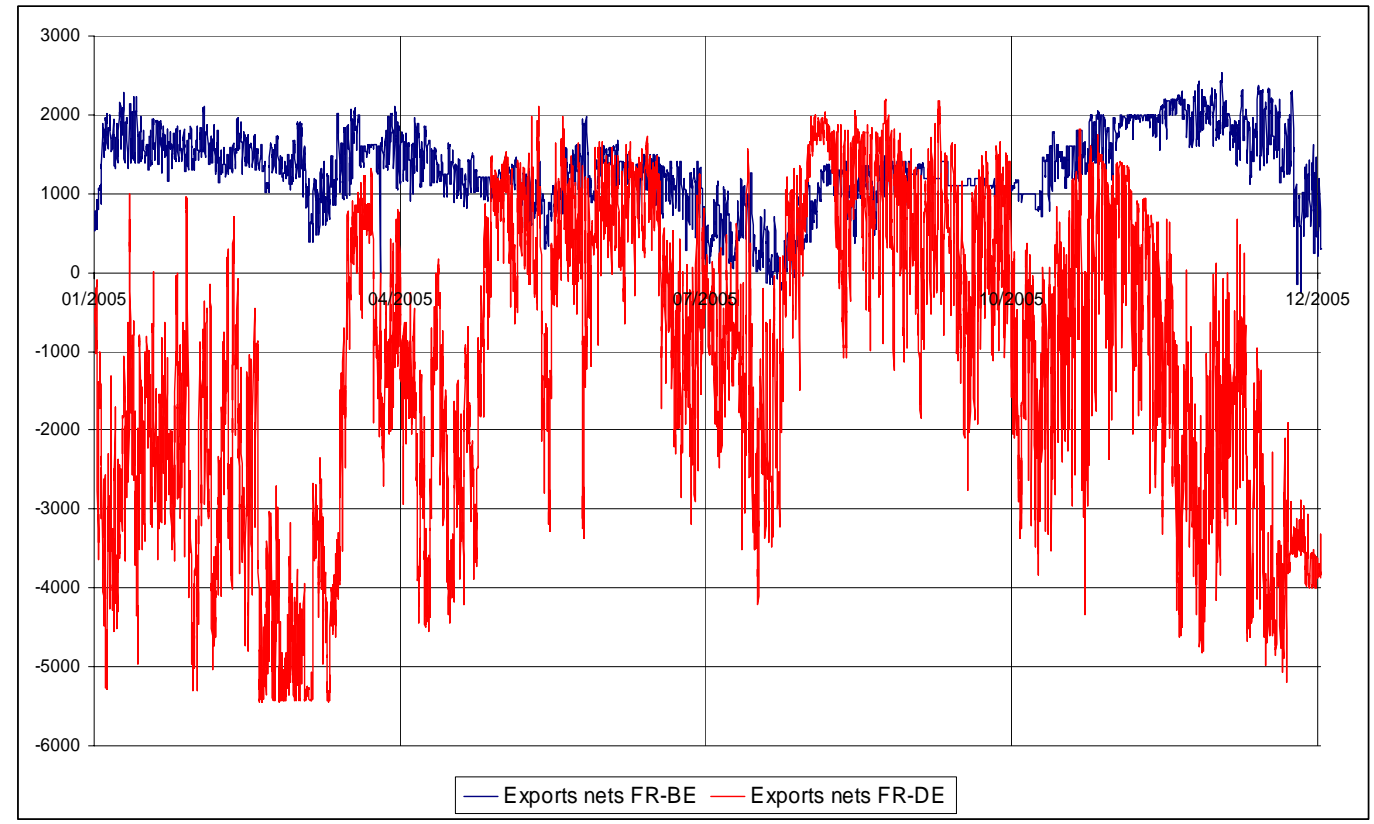

Echanges commerciaux nets (MW) France vers Belgique et France vers Allemagne en 2005

En partant de logiques relativement simples permettant de définir les capacités commerciales entre pays voisins en extrapolant des situations moyennes déterminées par des historiques, les mécanismes d'allocation transfrontaliers évoluent progressivement vers des logiques plus sophistiquées où ce sont in fine les prix que les acteurs associent à leurs demandes qui déterminent les directions de marché à privilégier. Cette évolution peut inclure le choix, pour les 
acteurs, entre des mécanismes de type option ou obligation ${ }^{1}$ et rapprochera en tout état de cause allocation des capacités transfrontalières et mécanismes de formation des prix de livraison physique dans chaque pays.

\section{Un GRT délivre un service d'accès à l'interconnexion}

Entre la décision d'échanger un bloc d'énergie à une échéance donnée entre deux acteurs de part et d'autre d'une frontière et sa réalisation physique, la complexité de mise en œuvre requiert un ensemble d'actions et de services bien définis à accomplir par les deux GRTs concernés.

En amont, on trouve les études et discussions inter-GRTs typiquement en plusieurs phases de gestion prévisionnelle, de l'annuel au J-1- permettant de définir la capacité de l'interconnexion disponible à un instant donné, compte tenu de la maintenance et des indisponibilités fortuites ou programmées des ouvrages de transport et de production.

Ensuite, le service d'acquisition des capacités qui permet à chaque acteur de présenter ses demandes puis d'être crédité des droits obtenus en fonction des modalités mises en place voir ci-après-. Lorsque le niveau de coordination avec le GRT correspondant a atteint un niveau suffisant, l'acquisition des droits pour « sortir du premier GRT et entrer dans le second» est unique, c'est le schéma qui tend à se généraliser. Pour un niveau de coordination plus faible, l'acteur est obligé d'acquérir des droits séparément auprès de chaque GRT, c'est en particulier le schéma qui a prévalu au début de la mise en place du marché. On peut enfin envisager un niveau de coordination renforcé entre N GRTs, tel que les acteurs puissent acquérir directement des droits à transférer des blocs d'électricité entre deux pays non voisins.

Après nominations par les acteurs des échanges d'énergie qu'ils souhaitent effectivement programmer pour les créneaux temporels concernés (par ex, la journée), les deux GRTs vérifient que ces nominations restent à l'intérieur des droits acquis, et se coordonnent, afin de s'assurer que le total des demandes d'accès en export est cohérent avec le total des demandes en import. Cela se traduit in fine par la fixation des programmes d'échanges horaires requis pour le bon fonctionnement du réseau interconnecté et en particulier du Réglage Secondaire Fréquence-Puissance (RSFP), traduction pratique des échanges commerciaux convenus entre acteurs. Le RSFP, automate centralisé permettant de rétablir la fréquence et de tenir une consigne de bilan import-export d'une zone de réglage, est un des éléments essentiels permettant d'assurer la sûreté d'exploitation du point de vue de l'équilibre offre-demande dans le système interconnecté de l'UCTE, tout en autorisant le confinement au niveau régional en cas de perturbation majeure dans un GRT.

Enfin, un service de garantie des transactions commerciales est mis en place, avec des modalités variées, en fonction des échéances et des produits concernés. Par exemple, si RTE doit réduire des droits acquis aux enchères sur une capacité, il

\footnotetext{
${ }^{1}$ Dans le cadre de mécanismes de type « option », un acteur peut acquérir un droit (en MW) d'utilisation future d'une capacité, qu'il devra confirmer, à une date donnée avant l'échéance, en "nominant » son programme (inférieur ou égal au niveau en MW de cette option) ; dans le cas d'une « obligation », la nomination est effectuée simultanément avec l'acquisition du droit de capacité et est définitive.
}

indemnise l'acteur à hauteur de $110 \%$ du prix payé pour l'acquisition. Lorsqu'une réduction de capacité intervient après l'échéance de nomination du $\mathrm{J}-1$, sous réserve de faisabilité technique, l'échange programmé est garanti, au prix d'actions de redispatching parfois très coûteuses.

\section{LES GRANDES CLASSES DE MODALITES D’ATTRIBUTION DES CAPACITES}

Jusqu'à aujourd'hui, tous les GRTs européens raisonnent en terme de volume d'échanges commerciaux entre pays voisins. Ce que nous appelons dans cet article allocation en volume correspond à un processus où il est admis que les GRTs définissent a priori des volumes d'échanges commerciaux à allouer entre pays voisins sur chaque chemin contractuel, puis que les marchés, en remplissant ces volumes, équilibrent les prix entre ces pays ou du moins les rapprochent jusqu'à buter sur les limites de sûreté du réseau. Le volume disponible sur un chemin contractuel compte tenu des limites de sûreté du réseau est appelé ATC (Available Transfer Capability). Par ailleurs, on utilise aussi la notion de NTC (Net Transfer Capability) qui définit la capacité totale disponible pour un jour donné (et dont la valeur exacte n'est connue qu'en J-1). Celle-ci va être allouée le plus souvent en plusieurs étapes successives, allant du moyen terme (annuel, trimestriel...) au court terme (J-1 principalement), la somme des ATC alloués à ces différentes étapes correspondant à la NTC.

Le choix des mécanismes d'allocation est de la responsabilité des régulateurs. Les GRTs, responsables de l'accès aux interconnexions, contribuent à la définition de ces mécanismes d'allocation etsont en charge de leur mise en œuvre. Nous décrivons ci-après succinctement les principaux mécanismes utilisables.

\section{A. Mécanismes administrés}

Lorsque les GRTs attribuent l'ATC suivant un mécanisme administré, chacun applique les règles édictées par son régulateur : liste de priorité, pro-rata des demandes, limitation des volumes allouables à un acteur... Dans ce cas, l'allocation est généralement gratuite. L'objectif souvent recherché dans les règles mises en place est de faciliter la fluidité d'accès aux interconnexions, pour les petits acteurs, et de réduire le pouvoir de marché des gros acteurs, par exemple en limitant le pouvoir de rétention de capacité non utilisée. Cependant, ces mécanismes laissent l'entier profit de l'utilisation de l'interconnexion aux quelques acteurs qui obtiennent des droits d'utilisation, acteurs qui bénéficient du différentiel de prix de marché entre les deux zones interconnectées, ce qui est discriminatoire vis à vis des autres clients des GRTs, et ne permet pas d'avoir de signaux économiques pertinents sur les directions de marché et sur l'intérêt d'augmenter les capacités. C'est la raison pour laquelle, le Règlement Européen 1228/2003 impose aujourd'hui l'abandon des mécanismes administrés et la généralisation de mécanismes de marchés pour l'attribution des droits de capacité, du moins en présence de risques de congestion.

\section{B. Enchères explicites}

Parmi ces mécanismes de marché, le plus naturel est de mettre ces droits aux enchères, on parle d'enchère explicite. Dans ce 
cas les GRTs de part et d'autre des frontières concernées organisent séparément ou en commun des enchères où sont attribuées les ATC. L'enchère a pour objet de donner les droits aux acteurs qui sont prêts à les payer les plus chers, et qui, de ce fait, doivent être les plus compétitifs. Mais si la capacité disponible est insuffisante pour satisfaire toute la demande, elle apporte aussi aux GRTs un revenu de congestion, qui sera utilisé, soit pour financer les actions éventuelles de redispatching nécessaires à la fermeté des échanges, soit pour investir ultérieurement ${ }^{2}$ de façon à augmenter les capacités de transport des corridors congestionnés soit pour diminuer les tarifs d'accès au réseau, ce qui bénéficie ainsi à l'ensemble des utilisateurs. Depuis que ces enchères ont vu le jour à différentes frontières en Europe, diverses caractéristiques techniques de ces enchères ont été utilisées afin de les rendre les plus compétitives possible, de réduire le pouvoir de marché, ... : multiplication des enchères, enchère à plusieurs tours ou à durée variable, paiement au prix d'offre ou marginal, mise en place d'un principe «Use It Or Lose It » (UIOLI : la capacité non utilisée à l'heure de la nomination des droits est perdue sans compensation et peut être réallouée à d'autres utilisateurs) entre les différentes étapes de nomination et allocation...

Ces droits attribués constituent en fait des réservations de capacités (dans la très grande majorité des cas), mais n'obligent pas l'acteur à les utiliser. En effet, c'est dans une étape ultérieure, et en dernier lieu après le marché spot J-1 -ou infra-journalier- que chaque acteur "nomine » sa position définitive sur l'interconnexion, et qu'il restitue ou non la part non utilisée, en fonction des règles mises en place.

Ces enchères explicites constituent un progrès majeur dans l'attribution des droits de capacité. Néanmoins, elles présentent des inconvénients, en particulier pour les échéances de court terme :

La séparation temporelle entre le marché des capacités et le marché de l'énergie (spot) qui lui succède oblige les acteurs à formuler leurs offres de prix pour la capacité en restant dans l'incertitude quant à leurs positions finales sur chacun des deux marchés et dans l'incertitude sur le différentiel de prix final. Ces risques pèsent naturellement sur le comportement économique des acteurs. On assiste donc parfois à des incohérences, par exemple quand les capacités sont valorisées de façon non négligeable dans les deux sens de l'interconnexion, ou encore lorsque les valorisations des capacités sont contradictoires avec le différentiel de prix court terme constaté in fine ${ }^{3}$.

Par ailleurs, l'absence de fermeté des droits acquis, qui ne sont en général que des options, interdit de

\footnotetext{
${ }^{2}$ Dans le cas des liaisons à courant continu dont le coût et mode d'exploitation se distinguent nettement du reste du réseau d'interconnexion, l'amortissement d'un investissement passé est également une raison pour mettre en œuvre des enchères.

${ }^{3}$ En fait, l'analyse est plus compliquée, car la valorisation consentie par un acteur à une date donnée pour une interconnexion résulte de l'ensemble de ses positions commerciales construites sur des contrats de long et moyen terme antérieurs à son enchère, de son anticipation des positions finales des marchés et des positions qu'il acquerra en court terme....
}

tenir compte en phase d'allocation du fait que les échanges de sens opposé se compensent (phénomène de superposition, dit «netting»). Ceci induit parfois une sous-utilisation potentielle de la capacité en phase de nomination.

Enfin, lorsqu'une partie des droits alloués n'est pas utilisée en $\mathrm{J}$-1, et ne peut être réallouée avant le temps réel, cela correspond à une perte d'efficacité économique globale.

\section{Enchères implicites}

Les GRTs peuvent combiner l'attribution des ATC avec le fonctionnement de bourses de l'électricité. On parle alors d'enchères implicites (par opposition aux enchères explicites) ou de couplage de marchés. Les GRTs calculent les ATC et concèdent aux bourses le service d'allocation de ces capacités à l'échéance des marchés spot journaliers, les GRTs restant responsables en tout état de cause de la gestion des échanges. Les acteurs peuvent ainsi profiter des opportunités de vente ou achat transfrontaliers simplement en présentant des offres prix-quantité sur au moins une des bourses des zones couplées. Ils n'ont plus à se soucier, antérieurement aux marchés spots, d'acquérir des droits de capacités, ni à fixer la zone de destination de leur offre. Les bourses de la région concernée font leur clearing en commun ou de façon itérative et il en résulte dans un premier temps un prix spot unique correspondant à l'ensemble des offres. Si la combinaison des offres concourrant à ce prix ne provoque pas de dépassement des ATC, les offres correspondantes sont retenues et le prix spot s'applique à toutes les zones. Si certaines ATC sont dépassées, les zones adjacentes sont séparées, une fourniture (resp. demande) non nominative égale à l'ATC est prise en compte dans la zone importatrice (resp. exportatrice) et les prix spots qui en résultent diffèrent d'une zone à l'autre.

Le couplage de marché délivre ainsi des obligations à produire ou à consommer dans telle ou telle zone de marché tandis que l'enchère explicite délivre des droits physiques autorisant à mettre en place un échange transfrontalier. On notera aussi que le couplage de marché génère un revenu de congestion du même ordre de grandeur que celui d'une enchère explicite, qui a vocation à être géré par les GRTs de façon similaire.

Les avantages majeurs de ce mécanisme d'attribution des capacités sont :

L'efficacité économique: par construction, la méthode utilisée maximise la valeur que les acteurs donnent à l'existence de l'interconnexion, puisque son utilisation finale, en volume et en direction, est directement liée aux valorisations de l'achat/vente d'énergie sur les marchés couplés. S'agissant d'obligations indirectes d'utilisation de la capacité, la superposition des offres dans les deux sens est prise en compte automatiquement ;

La simplicité pour les acteurs ;

La réduction du pouvoir de marché puisque aucun acteur ne peut acquérir de droits individuels avant le marché d'énergie, et encore moins les soustraire, puisque la concurrence sur chacun des marchés 
couplés augmente et puisque la formation des prix est transparente ;

L'augmentation de la liquidité des marchés ainsi couplés et leur meilleure résilience (sensibilité du prix de clearing à une offre supplémentaire) ;

La capacité de coupler par ce mécanisme plus de deux marchés, tout en gardant un fonctionnement parfaitement symétrique.

On notera qu'il existe deux principales variantes pour l'implémentation de ces enchères implicites avec couplage de marché, qui sont fonctionnellement identiques mais s'adaptent mieux aux particularismes locaux :

soit le «market coupling » (couplage de marchés) qui consiste en un couplage de $\mathrm{N}$ bourses selon une approche décentralisée résultant en un marché unique virtuel tant que les capacités d'interconnexion ne sont pas saturées,

soit le «market splitting » (fragmentation du marché) dans lequel un marché, unique au départ, est séparé en tant que de besoin en $\mathrm{N}$ marchés indépendants virtuels lorsque les capacités sont saturées.

La mise en œuvre sous forme de "market splitting " s'est développée depuis plusieurs années avec succès dans le cas de NordPool.

Quant à la mise en oeuvre sous forme de « market coupling », les gestionnaires de réseau français, belge et néerlandais, avec les bourses correspondantes Powernext, Belpex, APX, travaillent à la mettre en œuvre pour ces trois marchés en 2006, sous réserve de l'approbation des trois régulateurs concernés. C’est le projet « Tri-Lateral Coupling ».

\section{ETAT DES LIEUX POUR L'ACCES AUX INTERCONNEXIONS DE RTE}

L’année 2006 est caractérisée pour RTE par la généralisation de mécanismes de marchés sur les différentes frontières. L'approche définie par la CRE est assez pragmatique dans la mesure où un cadre général unique a été fixé, mais des adaptations particulières pour chaque frontière ont été faites, pour tenir compte de différents facteurs: historique des modalités d'accès antérieurs, organisation et régulation de chaque marché voisin... Il est fait usage tant des mécanismes explicites qu'implicites. Le paysage décrit ci-après évolue rapidement, aussi faut-il considérer cette description comme un instantané à la date de rédaction.

Par ailleurs, un changement important est intervenu fin 2005, la suppression par la CRE de la priorité des contrats long terme antérieurs à l'ouverture des marchés pour l'accès aux interconnexions avec les autres pays de l'UE. Dès lors les volumes de capacités attribuables via des mécanismes de marchés sont fortement augmentés.

Enfin, il faut souligner que RTE offre d'ores et déjà un service d'accès aux interconnexions en infra-journalier, via douze guichets (6 pour France/Espagne) permettant d'acquérir, lorsqu'elle est disponible, la capacité restée inutilisée.

\section{A. Grande-Bretagne - France}

L'interconnexion Angleterre-France a été la première frontière française sur laquelle une enchère explicite a été organisée pour l'allocation de droits de capacités de transport (2001).

Il n'y a aucune autre interconnexion entre la Grande-Bretagne et l'Europe continentale. De ce fait, il n'existe pas de flux en boucle lors de la détermination de la valeur de l'ATC de cette frontière.

Les enchères sont organisées conjointement par les deux GRT respectifs, NGT et RTE. Les produits vendus aux enchères sont les droits annuels, saisonniers, trimestriels, mensuels et journaliers pour le transfert de l'électricité dans le sens GB vers FR ou FR vers GB.

L'année 2005 a vu la mise en place de dispositions facilitant le marché secondaire en permettant aux acteurs ayant acquis des droits de notifier à RTE leur cession à d'autres.

\section{B. Belgique - France}

Comme on l'a vu, et sous réserve de l'approbation des trois régulateurs concernés, il est prévu la mise en place en 2006 sur cette frontière d'un mécanisme d'allocation très innovant, combinant les enchères coordonnées explicites aux horizons annuels et mensuels, avec application d'un principe de «Use it Or Lose it " après nomination, et un couplage des trois marchés spots français, belge et néerlandais à l'horizon $\mathrm{J}-1$, dénommé Tri Lateral Coupling (TLC).

Durant une phase transitoire de quelques mois, dans l'attente de l'approbation et de l'arrivée opérationnelle du TLC, on procédera à des enchères explicites journalières en J-1.

\section{C.Allemagne - France}

La capacité d'interconnexion entre ces deux pays est importante, dans les deux directions, aussi la capacité n'estelle pas toujours saturée. Néanmoins, il existe des situations contraintes où la saturation est atteinte. Aussi, en application des directives conjointes définies par les régulateurs français et allemands en 2005, des mécanismes d'enchères coordonnés sont mis en place début 2006, dans les deux directions, aux échéances annuelles, mensuelles et journalières.

\section{D.Italie - France}

Depuis le début de l'année 2005, les droits de transport de la France vers l'Italie ont été mis aux enchères par RTE sur une base mensuelle, limités à la moitié de la valeur de l'ATC. Ces droits, une fois attribués, sont fermes. La moitié restante est gérée par GRTN sous la forme d'enchères implicites, assorties d'un dispositif de couverture des positions à long terme par Droits de transport financiers (Financial Transmission Rights) pré-distribués.

En 2006, l'essentiel de ces principes reste d'actualité, cependant une enchère annuelle France/Italie a été introduite.

L'absence de congestion rend inutile la mise en place de mécanismes particuliers pour l'import, toutes les demandes raisonnablement envisageables compte tenu des différences structurelles des deux marchés pouvant être satisfaites sans congestion. 


\section{E. Suisse - France}

Stricto sensu, sur cette frontière, les directives de l'UE ne s'appliquent pas, puisque la Suisse n'en est pas membre. Ainsi, jusqu'en fin 2005, il n'existait pas de séparation des fonctions de gestion de réseau et de production/commercialisation d'énergie au sein des entreprises intégrées suisses. Les droits de capacité FranceSuisse, jusqu'à cette date, étaient pour l'essentiel attribués aux contrats long terme antérieurs à la mise en place du marché de l'électricité dans l'Union.

La Suisse étant en train de se doter d'un Opérateur de Système indépendant, il faut s'attendre à des évolutions des modalités mises en œuvre dans les mois à venir. En 2006, la mise en œuvre d'enchères journalières pour les capacités restant disponibles après nominations des contrats long terme (qui restent prioritaires sur cette frontière) devrait être effective.

\section{F. Espagne - France}

L'interconnexion Espagne-France est située dans une configuration de péninsule, ce qui simplifie la définition de la NTC entre les marchés. Cependant, l'architecture du marché en Espagne est très différente de celle de la France et, de ce fait, la coordination entre les opérateurs de réseau présente quelques difficultés. La différence la plus frappante entre les structures de marché est l'existence d'un marché de type pool physique en Espagne. Les activités du marché et du système électrique sont donc fortement liées l'une à l'autre. La logique naturelle serait donc de mettre en place un mécanisme de type implicite.

Dans un premier temps, en application des directives conjointes élaborées par les deux régulateurs début 2005, il est prévu en 2006 de mettre en place un système d'enchères explicites, devant évoluer à terme vers un couplage de marché pour l'allocation des capacités en J-1.

\section{V.PERSPECTIVES}

De nombreuses évolutions sont encore à venir pour ce qui concerne la gestion de l'accès aux interconnexions, et leurs conséquences sur le comportement des acteurs sur les différents marchés interconnectés. On peut en particulier citer celles-ci :

Le renforcement des services d'accès aux interconnexions en infra-journalier, permettant à chaque Responsable d'équilibre d'améliorer ses possibilités d'équilibrage et à chaque acteur d'optimiser les opportunités d'utilisation de ses actifs

On peut également penser que le développement des mécanismes implicites d'allocation ira croissant. Parallèlement, il est souhaitable aussi à terme de rendre possible la déclaration directe d'une transaction entre deux acteurs localisés dans des GRT non contigus.

Par ailleurs, la demande des acteurs de disposer d'outils toujours plus sophistiqués pour réduire leur exposition aux risques de fluctuation des marchés devrait conduire au développement progressif d'outils financiers de couverture, soit conservant un lien avec la capacité physique sous-jacente
(Financial Transmission Rights), soit purement financiers (Contracts for Differences utilisés sur le NordPool).

La mise en œuvre de ces évolutions ne sera néanmoins possible que dans la mesure où les cadres législatif et réglementaire en vigueur dans chaque pays continueront à converger pour être suffisamment compatibles, et où les GRTs poursuivront leurs efforts pour mieux coordonner leurs activités opérationnelles.

\section{RÉFÉRENCES}

"Congestion and risk management as seen by access clients or by TSO”, J-G Valentin, D.Aelbrecht, J-M Coulondre, P.Halain - CIGRE 2004, Paris.

"Managing cross-border congestion in a european market environment : the French case”, J-G Valentin, J-M Coulondre, J.Perez, D.Chaniotis - CIGRE-IEEE/PES 2005, New Orleans.

« Maîtriser les flux transfrontaliers, entre volume et valeur du marché », J-M Coulondre - REE - octobre 2004

«Flow-based Market Coupling - A Joint ETSO-EuroPEX Proposal for Cross-Border Congestion Management and Integration of Electricity Markets in Europe » - ETSO Septembre 2004

«An Overview of Current Cross-border Congestion Management Methods in Europe » - ETSO - Septembre 2004

« Definitions of Transfer Capacities in liberalised Electricity Markets » - ETSO - Avril 2001

«Mécanisme de gestion coordonné sur l'interconnexion France-Espagne » - CRE - Janvier 2005

«Feuille de route pour la gestion des congestions sur l'interconnexion France-Allemagne » - Bundesnetzagentur CRE - Septembre 2005

"Intégration régionale des marchés de l'électricité belge, français et néerlandais » - CRE-CREG-Dte - Décember 2005

« Décision de la Commission de régulation de l'énergie du $1^{\mathrm{er}}$ décembre sur la mise en œuvre des programmes de travail dénommés «feuilles de route » concernant les procédures d'allocation de capacités d'interconnexion en 2006 et la prise en compte de l'arrêt de la CJCE C-17/03 du 07/06/05 » - CRE - Décembre 2005 